\title{
Consequences of HIV/Syphilis Co-Infection on HIV Viral Load and Immune Response to Antiretroviral Therapy
}

\author{
Lina Fan* \\ Aiping Yu* \\ Defa Zhang \\ Ziyu Wang \\ Ping Ma
}

Department of Infectious Diseases, Tianjin Second People's Hospital, Nankai University, Tianjin, 300192, People's Republic of China

*These authors contributed equally to this work
Correspondence: Ping Ma

Department of Infectious Diseases,

Tianjin Second People's Hospital, Nankai

University, No. 7, Sudi Road, Naikai

District, Tianjin, 300192, People's

Republic of China

Tel +86-22-27468019

Email maping@tj.gov.cn
Introduction: Although syphilis is a frequent co-infection in patients with human immunodeficiency virus (HIV) infection, the influence of syphilis on immune response and virologic failure in HIV-infected patients following initiation of antiretroviral therapy (ART) is not well-defined.

Methods: A retrospective study was conducted at Tianjin Second People's Hospital to evaluate the prevalence of syphilis and immune status in 4171 ART-naïve patients. The study included patients who initiated ART between August 2009 and June 2019.

Results: The prevalence of syphilis was $40.1 \%$ in all ART-naïve patients and $42.5 \%$ in ARTnaïve men who have sex with men. HIV/syphilis co-infection was associated with higher virologic failure (odds ratio (95\% confidence interval): $1.30(1.04,1.63)$ ). Patients with HIV/ syphilis co-infection had lower median $\mathrm{CD}^{+} \mathrm{T}$ cell counts and $\mathrm{CD} 4 / \mathrm{CD} 8$ ratios at baseline. After initiation of ART, patients co-infected with HIV/syphilis had smaller increases in CD4 ${ }^{+}$ $\mathrm{T}$ cell counts and CD4/CD8 ratios than patients infected only with HIV. The rate of recurrence of syphilis or reinfection was $9 \%(n=128)$ during seven years of ART.

Conclusion: HIV/syphilis co-infection had a negative impact on immune recovery and antiretroviral effectiveness. RPR titer and HIV viral load should be monitored in patients coinfected with HIV/syphilis, especially in patients with high RPR titers.

Keywords: HIV/syphilis, co-infection, antiretroviral therapy, virologic failure, CD4 response

\section{Introduction}

Syphilis, a sexually transmitted infection caused by the bacterium Treponema pallidum, is frequently encountered amongst human immunodeficiency virus (HIV)-infected individuals. The prevalence of co-infection with HIV and syphilis varies from $8 \%$ to $25 \%$, ${ }^{1,2}$ depending on the prevalence of both infections within the community and the group of patients studied. In China, the prevalence of HIV and syphilis among men who have sex with men (MSM) was recently shown to be higher than that in heterosexual men (about $6.5 \%$ and $11.2 \%$, respectively). ${ }^{3}$ Although homosexual transmission has been recorded as the major route of transmission of HIV in Tianjin, ${ }^{4}$ there is no information about the prevalence of syphilis among HIV-infected patients, especially among HIV-infected MSM.

$\mathrm{HIV} /$ syphilis co-infection is considered to be a dangerous combination ${ }^{5}$ since HIV makes failure of syphilis treatment more likely ${ }^{6}$ and co-infection leads to more profound neurocognitive impairment. ${ }^{7}$ Although active antiretroviral therapy (ART) 
allows successful management of HIV infection and enhances the lives of people living with HIV/AIDS (PLWHA), the consequent downregulation of innate and acquired immune responses to syphilis may increase susceptibility to infection. ${ }^{8}$ Reciprocally, syphilis enhances the likelihood of HIV transmission ${ }^{9}$ since it can negatively impact the immune system, and has been shown to increase HIV viral loads and reduce $\mathrm{CD}^{+} \mathrm{T}$ cell counts in PLWHA receiving ART. ${ }^{10-14}$ The relationship between HIV/syphilis co-infection and treatment responses after initiation of ART has not been fully investigated. Thus, the aim of this study was to evaluate the impact of syphilis on the virologic (HIV viral load) and immunologic (CD4 counts, CD4/CD8 ratio) parameters of PLWHA, before and after initiation of ART.

\section{Methods}

\section{Study Population}

The study was conducted at Tianjin Second People's Hospital, China, which, since August 1999, has been a designated hospital for the treatment of HIV. The study methodology conformed to the Declaration of Helsinki and was approved by the ethics committee of Tianjin Second People's Hospital. HIV positive patients initiating ART in 2009 and followed up until June 2019 were considered for inclusion in the study. After authorization by the ethics committee (Approval no. 2019-7), available patient data were provided from electronic medical records by the hospital and the need for informed consent was waived.

From the available data, we collected information about 4171 patients who initiated ART from 2009 and were followed up until 2019. After applying exclusion criteria, incomplete information (ie, individuals without examinations for syphilis before antiretroviral treatment initiation, $\mathrm{n}=347$ ) were excluded. Indeed, to investigate the consequences of HIV/syphilis coinfection on HIV viral load and immune response to antiretroviral therapy, 3829 PLWHA were followed up after the initiation of ART. CD4 lymphocyte counts and viral loads were determined according to clinical protocols: assessments were initially performed 6-monthly, then yearly or twice per year after the first year on ART from 2009. The exclusion criteria included fatalities $(n=59)$ and syphilis diagnosed after ART initiation $(n=92)$. Of the remaining 3678 patients initiating ART who were included in the study, 2164 had only HIV infection and 1514 were co-infected with syphilis. A flow chart showing patient characteristics and selection is provided in Figure 1A.

\section{Follow-Up}

As shown in Figure 2A, two patients were infected with only HIV in 2009, and one of whom was removed from the analysis due to the diagnosis of syphilis after ART initiation, and the other was deceased. In 2010, 10 patients with monoHIV infections were included, of whom, three patients were removed from the analysis due to the diagnosis of syphilis after ART initiation, two were deceased, and five began ART after July 2010. Thus, no data from participants with mono-HIV infections were included in 9-year follow-up analysis. Similarly, no such data were used for the 8-year follow-up analyses for the same reasons. As a result, patients were followed up with for up to 7 years.

\section{Definition}

HIV virologic failure was defined as two consecutive measurements with HIV RNA $>200$ copies/mL after six months of ART. ${ }^{15}$ Recurrent syphilis or reinfection were identified by a 4-fold increase in antibodies in a Rapid Plasma Reagin (RPR) test ${ }^{16}$ following a previously documented successful course of treatment for syphilis and were evaluated by a specialist in sexually transmitted infections.

\section{Data Collection}

Retrospective data, including demographic and clinical/ laboratory characteristics, were collected from medical records. Variables included age, sex, HIV transmission route, co-infection with hepatitis B virus (HBV), coinfection with hepatitis $\mathrm{C}$ virus (HCV), HIV stage as defined by the World Health Organization (WHO), ${ }^{17}$ HIV-1 viral load and body mass index (BMI). CD4 $4^{+}$ T cell count was stratified as $<50$ cells $/ \mu 1,50-200$ cells/ $\mu \mathrm{L}$ and $>200$ cells $/ \mu 1 .{ }^{18} \mathrm{HIV}$ viral load at baseline was not included in the medical records, and therefore, based on WHO clinical criteria for HIV disease in adults and adolescents, subjects were classified into stage I, stage II, stage III and stage IV infection. ${ }^{17}$ BMI was stratified as underweight $\left(<18.5 \mathrm{~kg} / \mathrm{m}^{2}\right)$, normal weight $(18.5-24 \mathrm{~kg} /$ $\left.\mathrm{m}^{2}\right)$ and overweight and obesity $\left(>24.0 \mathrm{~kg} / \mathrm{m}^{2}\right) .{ }^{19,20}$

\section{Statistical Analysis}

Statistical analyses were performed using SPSS 22.0 software (SPSS, Chicago, IL, USA) and GraphPad 7 (GraphPad Software, La Jolla, CA, USA). Because of skewed statistical distributions, numerical data are presented as medians with interquartile ranges; categorical variables are presented as percentages. Differences between two groups were analyzed 
A

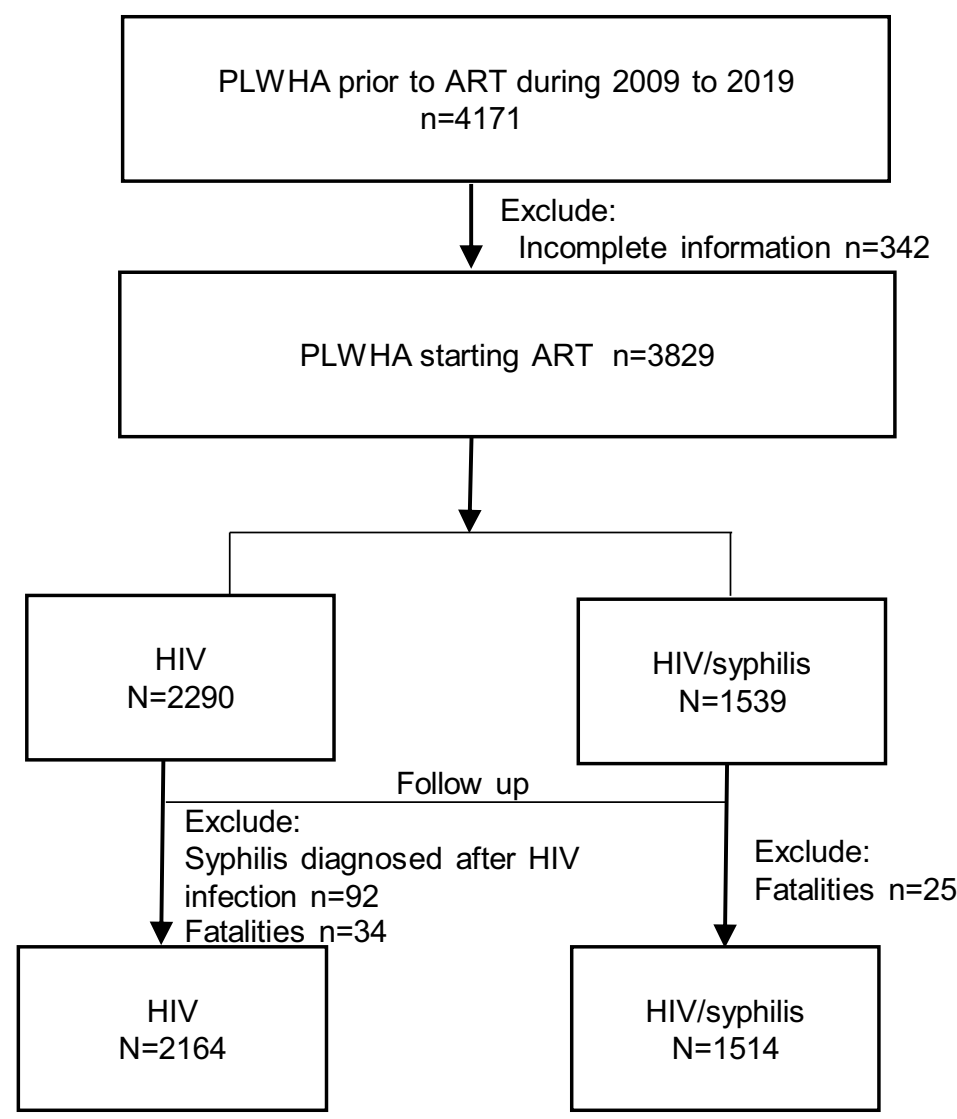

B

Syphilis-coinfection

1439

\section{$73 \quad 324$ \\ $82 \quad 5 \quad 38$}

HBV-coinfection HCV-coinfection

Figure I (A) Flow chart showing patient characteristics and selection; (B) Venn diagram showing co-infections in PLWHA. 
A

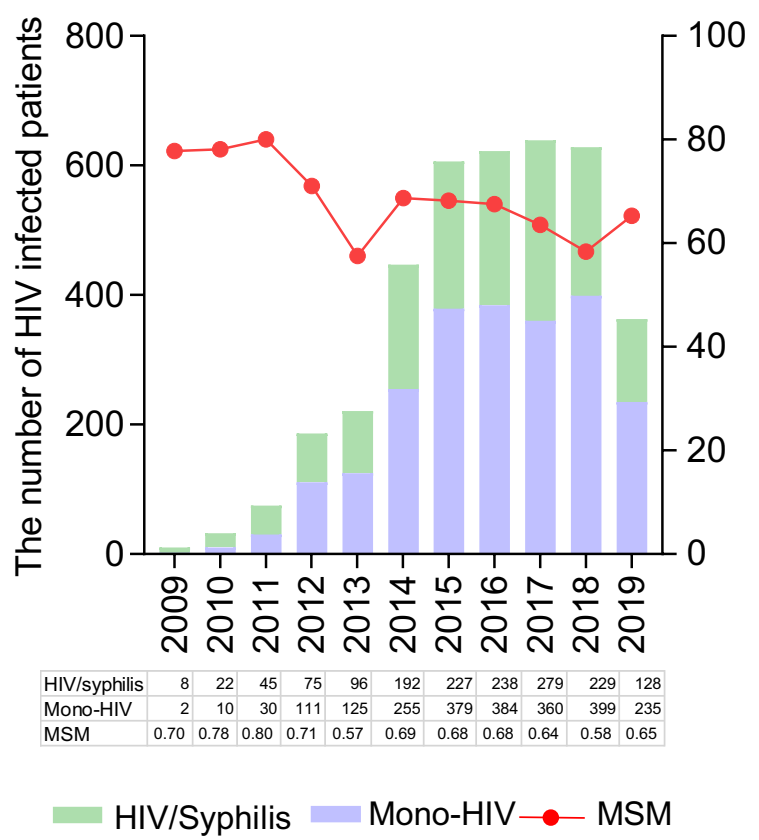

B

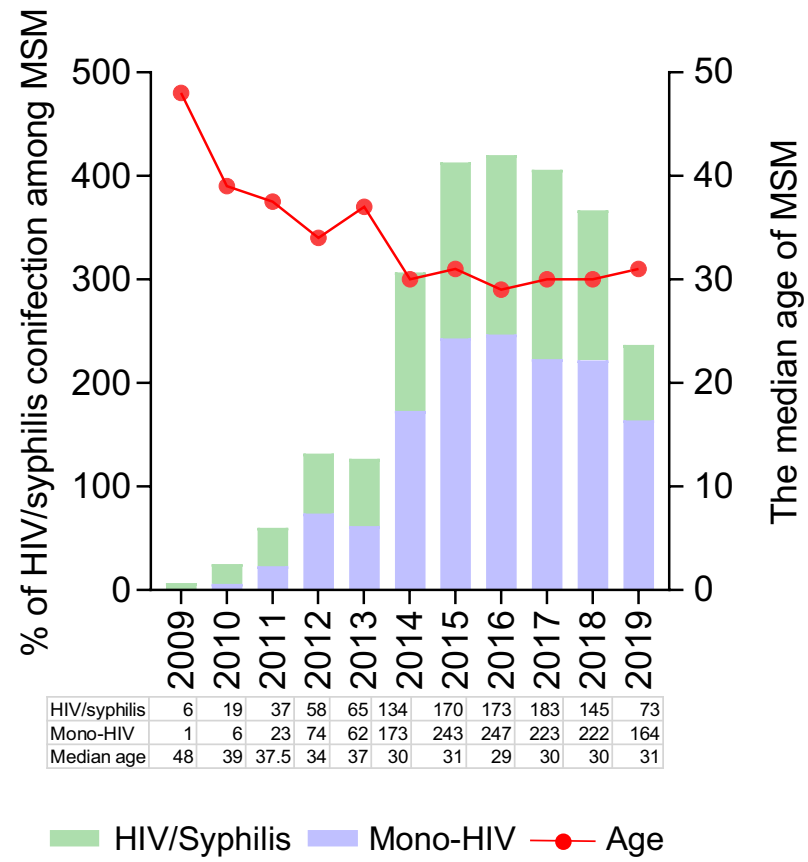

Figure 2 (A) Percentage of syphilis-positive cases and numbers of MSM among HIV-infected patients per year; (B) Annual syphilis rates and age in MSM with HIV infection.

using the Mann-Whitney $U$-test (nonparametric). Repeated measurements of $\mathrm{CD}^{+}{ }^{+} \mathrm{T}$ cell counts, $\mathrm{CD} 8^{+} \mathrm{T}$ cell counts and CD4/CD8 ratios during follow-up were modeled using linear mixed effects models, with a random subject effect. Binary logistic regression models were used to evaluate risk factors associated with virologic failure during seven years of ART. Kaplan-Meier curves were computed for this cohort. Log rank testing was carried out to evaluate differences in cumulative virologic failure in HIV-infected patients, with or without co-infection with syphilis. Demographic variables (sex, age), clinical/laboratory characteristics $\left(\mathrm{CD}^{+} \mathrm{T}\right.$ cell count, HIV stage, co-infection with syphilis, co-infection with HBV and co-infection with HCV) and the combination of antiretroviral regimens were included to investigate risk factors of virologic failure, regardless of their significance between groups at baseline. A $p$-value $<$ 0.05 was considered to be statistically significant.

\section{Results}

Demographic and Clinical Characteristics, and Prevalence of Syphilis in HIV Infected Patients Prior to ART

A total of 3829 individuals were included in the study, $1539(40.2 \%)$ of whom were co-infected with syphilis before initiation of ART (Figure 1A). The distribution of co-infection with syphilis, HBV and HCV at baseline is shown in Figure 1B. The median age of the study participants was 32 years, the ratio of men to women was 18.5:1, the median BMI was 22.2 (interquartile range [IQR], 20.1, 24.5) $\mathrm{kg} / \mathrm{m} 2$ and the median $\mathrm{CD} 4+\mathrm{T}$ cell count was estimated to be 268 (IQR, 145, 392) cells/ $\mu \mathrm{L}$. Same-sex relationships were the main route of transmission and accounted for $65.3 \%$ of all cases. Most of the participants (83.1\%, 3182/3829) had stage II HIV-1 infection, based on WHO criteria. Patients who were co-infected with syphilis were older (median age 34 vs 31 years, $p<$ $0.001)$ and more likely to be male ( $98.2 \%$ vs $92.8 \%, p<$ 0.001 ) than patients infected only with HIV. HIV/syphilis co-infection was also associated with lower $\mathrm{CD}^{+} \mathrm{T}$ cell counts (258 vs $276, p=0.01)$ and lower CD4/CD8 ratios ( 0.22 vs $0.25, p<0.001$ ) (Table 1$)$. The proportion of cases of HIV/syphilis co-infection decreased from $80 \%$ to $30 \%$ during the follow-up period of the study (2009 to 2019), although the homosexual transmission route remained steady at around 50-70\% (Figure 2A). Both the fraction of MSM co-infected with HIV/syphilis and the median age of MSM fell from 2009 to 2019 (Figure 2B). 
Table I Characterization of PLWHA Prior to ART

\begin{tabular}{|c|c|c|c|c|}
\hline Characteristics & Total $(n=3829)$ & Mono-Infected HIV $(n=2290)$ & HIV/Syphilis $(n=1539)$ & $P$ values \\
\hline Age (Year) & $32(26,44)$ & $31(25,42)$ & $34(28,46)$ & $<0.001$ \\
\hline Male & 3637 (95\%) & $2126(92.8 \%)$ & I5II (98.2\%) & $<0.001$ \\
\hline HBV co-infection & $162(4.2 \%)$ & 87 (3.8\%) & 75 (4.9\%) & 0.11 \\
\hline HCV co-infection & $70(1.8 \%)$ & $43(1.9 \%)$ & $27(1.8 \%)$ & 0.78 \\
\hline BMI $\left(\mathrm{kg} / \mathrm{m}^{2}\right)$ & $22.2(20.1,24.5)$ & $22.2(20.1,24.5)$ & $22.3(20.2,24.6)$ & 0.53 \\
\hline $\begin{array}{l}\text { Infection route } \\
\text { Heterosexual } \\
\text { Homosexual } \\
\text { Other }\end{array}$ & $\begin{array}{l}716(18.7 \%) \\
2501(65.3 \%) \\
612(16 \%)\end{array}$ & $\begin{array}{l}475(20.7 \%) \\
1438(62.8 \%) \\
377(16.5 \%)\end{array}$ & $\begin{array}{l}24 \mid(15.7 \%) \\
1063(69.1 \%) \\
235(15.3 \%)\end{array}$ & $<0.001$ \\
\hline $\begin{array}{l}\text { HIV stage } \\
\text { I } \\
\text { II } \\
\text { III } \\
\text { IV }\end{array}$ & $\begin{array}{l}363(9.5 \%) \\
3182(83.1 \%) \\
151(3.9 \%) \\
133(3.5 \%)\end{array}$ & $\begin{array}{l}205(9 \%) \\
1930(84.3 \%) \\
79(3.4 \%) \\
76(3.3 \%)\end{array}$ & $\begin{array}{l}158(10.3 \%) \\
1252(81.4 \%) \\
72(4.7 \%) \\
57(3.7 \%)\end{array}$ & 0.09 \\
\hline \multicolumn{5}{|l|}{ Laboratory data } \\
\hline CD4 (cells $/ \mu \mathrm{L})$ & $268(145,392)$ & $276(151,396)$ & $258(140,378)$ & 0.01 \\
\hline CD8 (cells/ $\mu \mathrm{L})$ & $1038(693,1458)$ & $1028(680,144 \mid)$ & $1059(707,1477)$ & 0.17 \\
\hline CD4/CD8 ratio & $0.24(0.14,0.37)$ & $0.25(0.14,0.38)$ & $0.22(0.13,0.35)$ & $<0.001$ \\
\hline $\begin{array}{l}\text { ART drugs } \\
\text { IN based } \\
\text { PI based } \\
\text { NNRTI based }\end{array}$ & $\begin{array}{l}175(4.6 \%) \\
494(12.9 \%) \\
3160(82.5 \%)\end{array}$ & $\begin{array}{l}112(4.9) \\
306(13.4 \%) \\
1872(81.7 \%)\end{array}$ & $\begin{array}{l}63(4.1 \%) \\
188(12.2 \%) \\
1288(83.7 \%)\end{array}$ & 0.27 \\
\hline
\end{tabular}

Note: Evaluation of median based on Q2 (QI-Q3).

Abbreviations: PLWHA, patients living with HIVIAIDS; IN, integrase inhibitor; NNRTI, non-nucleotide reverse transcriptase inhibitor; PI, protease inhibitor; ART, antiretroviral therapy.

\section{HIV/Syphilis Co-Infected Individuals Have Higher Risk of Virologic Failure During ART}

In the HIV/syphilis group, 63 patients received INR-based ART drugs, 188 received PI-based ART drugs, and 1288 received NNRI-based ART drugs. No significant differences were found between HIV/syphilis coinfection and HIV mono infection.

The effect of syphilis on virologic response to ART was assessed by plotting Kaplan-Meier survival curves (Figure 3). Log rank testing showed a significant difference between patients infected only with HIV-1 and patients co-infected with HIV/syphilis $(p=0.005)$.

The multivariate logistic regression model showed that HIV/HCV co-infection (adjusted odds ratio (AOR): 2.34, 95\% confidence interval (CI): 1.25-2.11, $p=0.01)$, age (AOR: 1.01, 95\% CI: 1.00-1.02, $p=0.046$ ), HIV/syphilis co-infection (AOR: $1.30,95 \%$ CI: $1.04-1.63, p=0.02$ ), HIV-1 stage III-IV (AOR: 1.89, 95\% CI: 1.31-2.74, $p<$ 0.001 ) and $\mathrm{CD}^{+} \mathrm{T}$ cells counts $<50$ cells $/ \mu \mathrm{L}$ (AOR1.89, 95\% CI: $1.31-2.02, p=0.001)$ were associated with increased virologic failure in Chinese HIV-1 infected patients (Table 2).

\section{HIV/Syphilis Co-Infected Individuals Have Poorer Immune Recovery During ART} CD4 is the main immunologic marker of HIV/AIDS progression and the $\mathrm{CD} 4 / \mathrm{CD} 8$ ratio is considered to be a marker for the risk of non-AIDS diseases. ${ }^{21}$ At baseline, HIV/syphilis co-infected subjects had, on average, slightly lower $\mathrm{CD}^{+} \mathrm{T}$ cell counts and lower CD4/CD8 ratios $(p=$ 0.01 and $p<0.001$, respectively, Table 1 ). Before initiation of ART, $\mathrm{CD}^{+} \mathrm{T}$ cell counts were higher in HIV/syphilis co-infected patients than in patients infected with only 


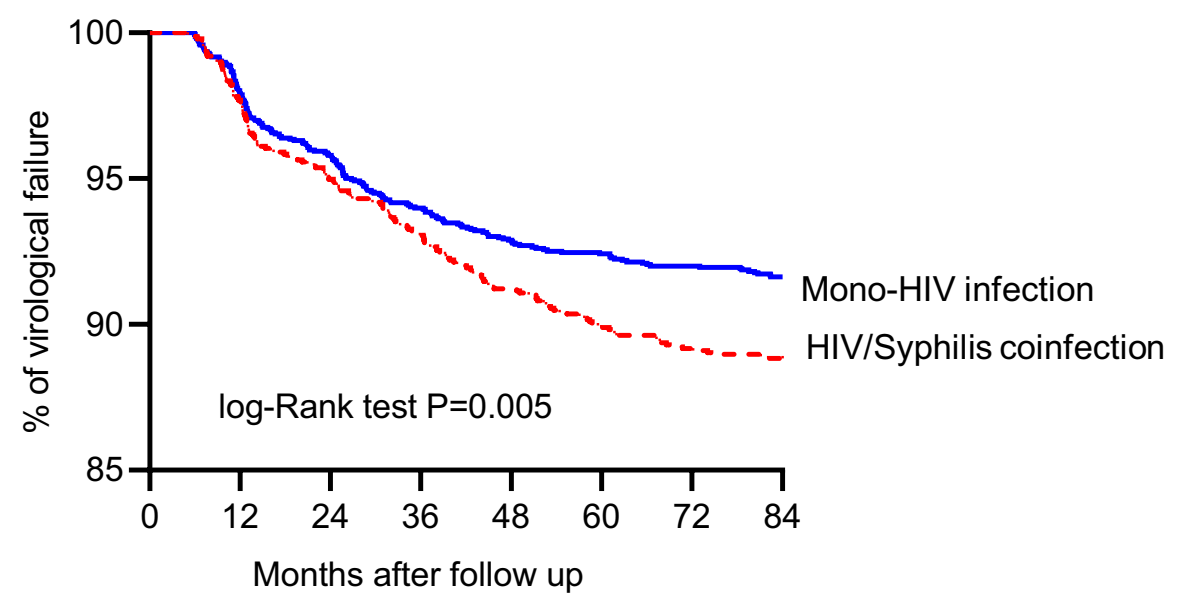

Number at risk by time

$\left.\begin{array}{r}\text { Mono-HIV } \\
\text { HIV/syphilis- }\end{array}\right]$\begin{tabular}{ccccccccc}
2164 & 1169 & 1078 & 782 & 513 & 294 & 125 & 39 \\
1514 & 861 & 744 & 587 & 383 & 249 & 106 & 37 \\
\hline & 12 & 12 & 24 & 36 & 48 & 60 & 72 & 84
\end{tabular}

Figure 3 Seven-year virologic failure curves of HIV infected patients with or without syphilis.

HIV, but the difference was not statistically significant ( $p=$ 0.17, Table 1). Individuals co-infected with HIV/syphilis had significantly slower recovery of $\mathrm{CD}^{+} \mathrm{T}$ cells and CD4/CD8 ratio compared with patients infected with only HIV (Figure 4), and the differences were significant after adjusting for baseline $\mathrm{CD} 4^{+} \mathrm{T}$ cell counts and other baseline covariates in the regression analyses (Table 3).

\section{Recurrence of Syphilis or Reinfection During Seven-Year Follow-Up}

The impact of recurrence or reinfection of syphilis on immune recovery and virologic failure during HIV treatment was also investigated. A total of 1423 patients were included in the analysis. Episodes of recurrence or reinfection with syphilis were estimated to be $9 \%(n=128)$ during the study period, and of which, at baseline, the proportion was $23.8 \%$ (31 cases) in patients with RPR titers $>1: 32$, which is significantly higher compared to $10.75 \%$ (57 cases) in patients with RPR titers 1:2-1:16 groups, and $6 \%$ (40 cases) in patients with RPR titers $<1: 2$ $(p<0.01)$ (Figure 5A). This indicated that high RPR titers at baseline were associated with a higher incidence of recurrence of syphilis or reinfection. $\mathrm{CD}^{+} \mathrm{T}$ cell counts and $\mathrm{CD} 4 / \mathrm{CD} 8$ ratios during the one-year periods before or after diagnosis in HIV/syphilis co-infection, and with or without the recurrence or reinfection with syphilis, are shown in Figure 5B and C. The difference between the two groups was not significant.

\section{Discussion}

In the present study, we found that individuals co-infected with HIV/syphilis before initiation of ART had a higher probability of virologic failure and smaller increases in $\mathrm{CD}^{+} \mathrm{T}$ cell count and CD4/CD8 ratios during seven years of ART, compared with individuals infected only with HIV. Higher RPR titers were associated with recurrence or reinfection with syphilis.

Syphilis is known to have a negative impact on immune recovery during HIV infection ${ }^{11}$ and our results are consistent with previous studies. As well as the change in $\mathrm{CD}^{+} \mathrm{T}$ cell counts, we observed, for the first time, that $\mathrm{CD} 4 / \mathrm{CD} 8$ ratios were reduced in these individuals at baseline and after seven years of ART. The CD4/CD8 ratio is a strong marker of immune activation and immune senescence, ${ }^{22,23}$ and a lower $\mathrm{CD} 4 / \mathrm{CD} 8$ ratio is also 
Table 2 Risk Factors for Virologic Failure in PLWHA During Seven Years of ART

\begin{tabular}{|c|c|c|c|c|c|}
\hline Variables at Baseline & df & Univariate Analysis OR $(95 \% \mathrm{Cl})$ & $p$ value & Multivariate Analysis OR $(95 \% \mathrm{Cl})$ & $p$ value \\
\hline Age & 1 & $1.01(1.00,1.02)$ & 0.02 & $1.01(1.00,1.02)$ & 0.046 \\
\hline HCV coinfection & 1 & & & & \\
\hline No & & Ref & - & Ref & - \\
\hline Yes & & $2.74(1.43,5.24)$ & 0.02 & $2.34(1.25,2.11)$ & 0.01 \\
\hline $\mathrm{CD} 4^{+} \mathrm{T}$ cell counts & 2 & & 0.001 & & 0.004 \\
\hline$\geq 200$ & & Ref & - & Ref & - \\
\hline $50-199$ & & $1.42(0.99,2.04)$ & 0.06 & $1.37(0.96,1.97)$ & 0.08 \\
\hline$<50$ & & $1.63(1.25,2.12)$ & 0.001 & $1.89(1.31,2.74)$ & 0.001 \\
\hline Syphilis & I & & & & \\
\hline No & & Ref & - & Ref & - \\
\hline Yes & & $1.28(1.02,1.61)$ & 0.03 & $1.30(1.04,1.63)$ & 0.02 \\
\hline WHO stage & I & & & & \\
\hline I-II & & Ref & - & Ref & - \\
\hline III-IV & & $1.91(1.32,2.76)$ & $0.00 \mathrm{I}$ & $1.89(1.31,2.74)$ & 0.001 \\
\hline ART drugs & 2 & & 0.20 & & 0.11 \\
\hline PI based & & Ref & - & Ref & - \\
\hline IN based & & $1.27(0.90,1.82)$ & 0.18 & $1.29(0.90,1.84)$ & 0.16 \\
\hline NNRTI based & & $0.76(0.37,2.76)$ & 0.45 & $0.73(0.36,1.52)$ & 0.40 \\
\hline HBV co-infection & I & & & & \\
\hline No & & Ref & - & & \\
\hline Yes & & $0.79(0.44,1.44)$ & 0.45 & & \\
\hline Sex & I & & & & \\
\hline Female & & Ref & - & & \\
\hline Male & & $1.04(0.59,1.85)$ & 0.89 & & \\
\hline
\end{tabular}

Abbreviations: IN, integrase inhibitor; NNRTI, non-nucleotide reverse transcriptase inhibitor; PI, protease inhibitor; ART, antiretroviral therapy; OR, odd ratio.

considered to indicate the presence of residual HIV viremia in virologically suppressed patients. ${ }^{24,25}$ Taken together, these findings allow us to speculate that coinfection with syphilis contributes to poor immune recovery and subsequently increases the likelihood of virologic failure.

An interesting finding of the present study is the higher probability of virologic failure in HIV/syphilis co-infected patients than in patients infected only with HIV during the seven-year ART follow-up period. To the best of our knowledge, this is the first evidence linking HIV/syphilis co-infection to virologic failure. Co-infection with syphilis could enhance HIV replication by increasing activation of host immune cells, altering the secretion of cytokines, including TNF- $\alpha$, and upregulating transcription factors, such as nuclear factor kappa beta. ${ }^{26}$

It has been shown that higher RPR titers are associated with an effective immunological response, leading to more effective elimination of T. pallidum. ${ }^{27}$ Previous studies have also shown that high RPR titers are associated with a good response to treatment for syphilis. ${ }^{28}$ In HIVinfected individuals, however, RPR titers $>1: 32$ have also been associated with laboratory-defined neurosyphilis. ${ }^{29}$ In the present study, we demonstrated a correlation between high RPR titers (>1:32) and recurrence or reinfection with syphilis. Further research is needed to investigate the incidence of neurosyphilis in recurrent syphilis and following reinfection. These results underline the importance of monitoring RPR titers in PLWHA co-infected with syphilis, even if these individuals have a good response to treatment, and this should be recognized by clinicians.

Since ART became widely available, the evidence that undetectable HIV equals non-transmissible HIV has spread, and the prevalence of HIV/syphilis co-infection has been significantly increased by the reduced use of condoms. ${ }^{8}$ Syphilis seroprevalence in individuals 


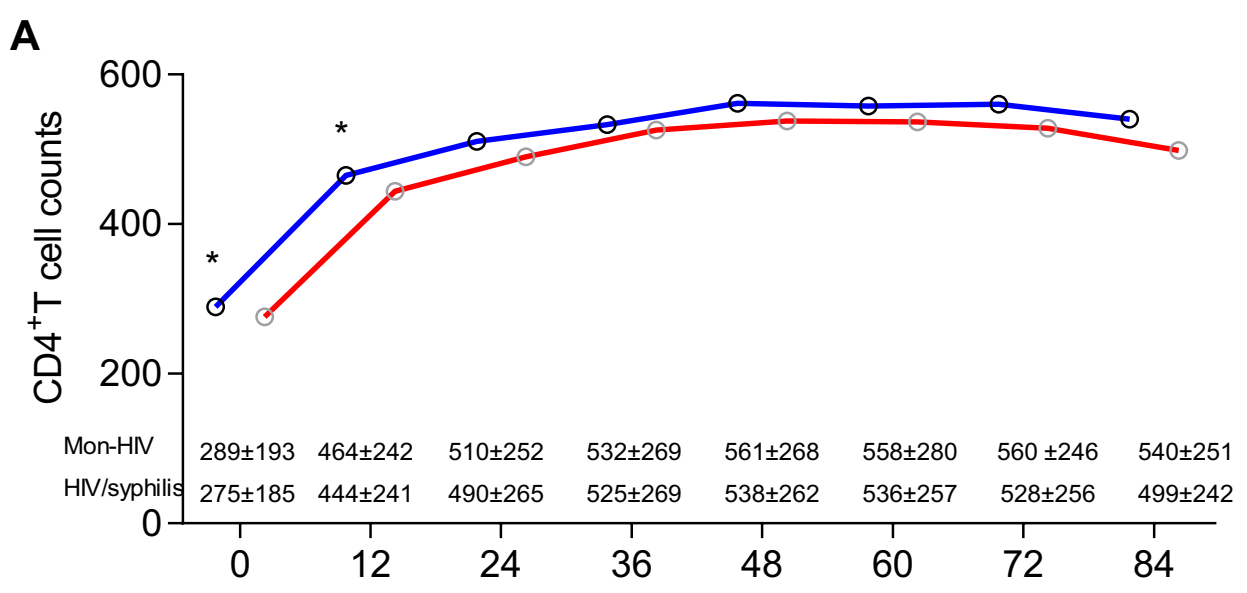

B
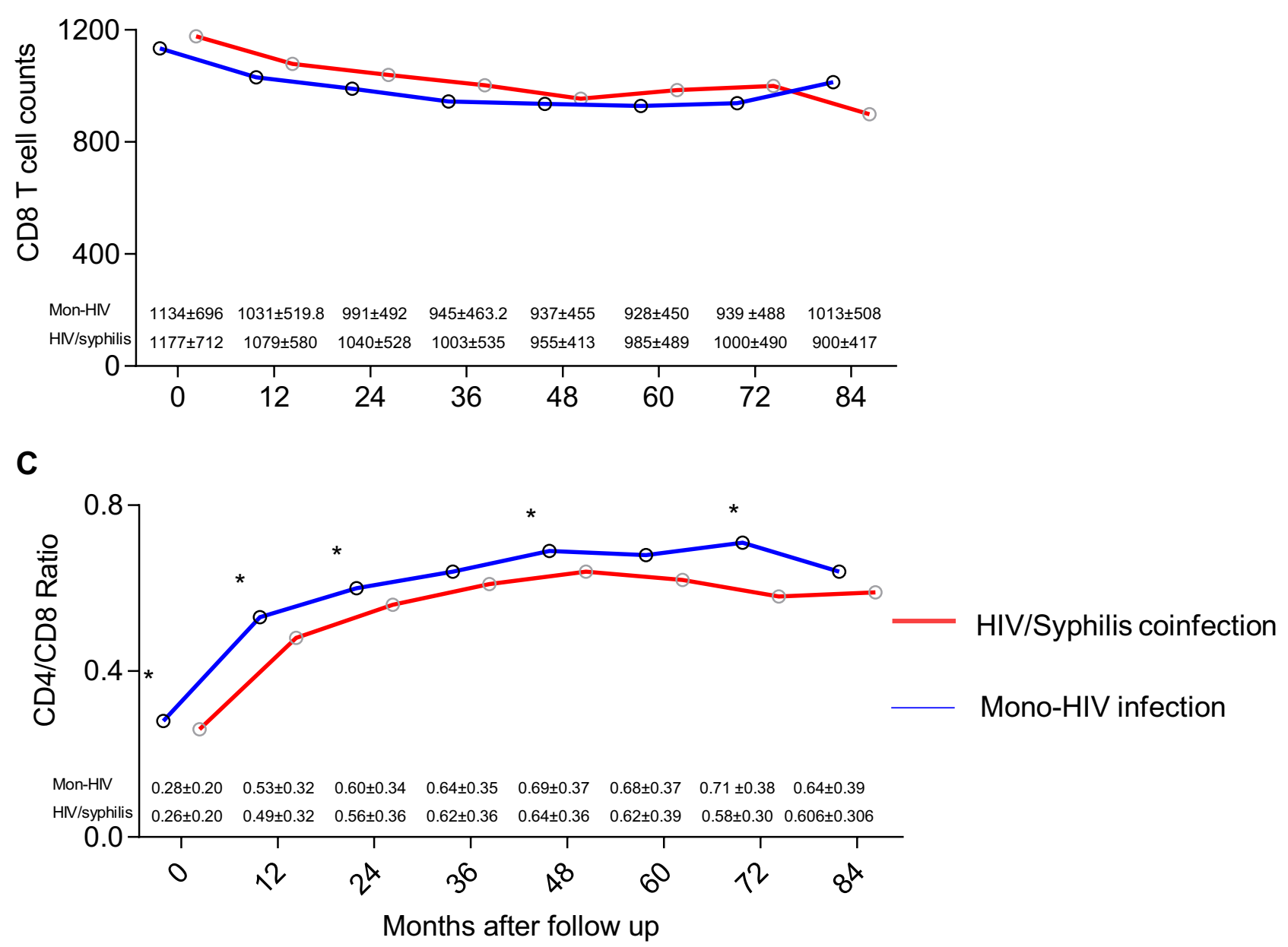

Figure 4 Trends of mean (A) CD4 ${ }^{+} \mathrm{T}$ cell counts, $(\mathbf{B}) \mathrm{CD} 8^{+} \mathrm{T}$ cell counts and (C) CD4/CD8 ratio, during seven-year ART follow-up according to syphilis status. ${ }^{*} p<0.05$. 
Table 3 Multivariable/Adjusted Models for Mean Differences in CD4 ${ }^{+}$T Cell Counts, CD8 ${ }^{+}$T Cell Counts and CD4/CD8 Ratios During Seven-Year ART Follow-Up

\begin{tabular}{|c|c|c|c|c|c|c|}
\hline \multirow[t]{2}{*}{ Variables } & \multicolumn{2}{|l|}{ CD4 } & \multicolumn{2}{|l|}{ CD8 } & \multicolumn{2}{|c|}{ CD4/CD8 } \\
\hline & Estimate $(95 \% \mathrm{Cl})$ & $p$ value & Estimate $(95 \% \mathrm{CI})$ & $p$ value & Estimate $(95 \% \mathrm{CI})$ & $p$ value \\
\hline Intercept & $301.61(275.05,328.17)$ & $<0.001$ & $968.8(904.69,1032.93)$ & $<0.001$ & $0.39(0.35,0.42)$ & $<0.001$ \\
\hline Syphilis/HIV co-infection at baseline & $-12.61(-22.57,-2.66)$ & 0.01 & $38.05(14.10,62.01)$ & 0.02 & $-0.03(-0.05,-0.02)$ & $<0.001$ \\
\hline HCV co-infection at baseline & $-47.08(-82.29,-11.87)$ & 0.01 & $-30.1(-83.55,23.29)$ & 0.27 & $-0.06(-0.11,-0.01)$ & 0.02 \\
\hline HBV co-infection at baseline & $9.98(-12.50,32.45)$ & 0.38 & $-30.12(-83.54,23.29)$ & 0.27 & $0.02(-0.01,0.05)$ & 0.21 \\
\hline Male & $7.20(-15.7930 .20)$ & 0.61 & II $9.66(64.78,174.53)$ & 0.001 & $-0.05(-0.08,0.02)$ & 0.04 \\
\hline \multicolumn{7}{|l|}{ ART drugs } \\
\hline IN based & $-46.6 \mathrm{I}(-84.95,-8.28)$ & $<0.001$ & $223.35(121.23,325.46)$ & $<0.001$ & $-0.12(-0.18,-0.06)$ & $<0.001$ \\
\hline NNRTI based & $-42.92(-58.46,-27.38)$ & 0.02 & $-90.46(-128.48,-52.45)$ & $<0.001$ & $-0.01(-0.03,-0.02)$ & 0.58 \\
\hline PI based & 0 & & 0 & & 0 & \\
\hline Baseline $\mathrm{CD} 4^{+} \mathrm{T}$ cell counts & $0.86(0.86,0.91)$ & $<0.001$ & $-0.10(-0.17,-0.02)$ & 0.005 & $0.03(0.05,0.02)$ & $<0.001$ \\
\hline
\end{tabular}

Abbreviations: IN, integrase inhibitor; NNRTI, non-nucleotide reverse transcriptase inhibitor; PI, protease inhibitor; ART, anti-retroviral therapy.

diagnosed with HIV has been reported to range from $5.2 \%$ to $25 \% .^{30-33}$ In the present study, at baseline, syphilis infection among PLWHA accounted for $40.2 \%$ of total infections. The reason for the higher prevalence was related to the higher proportion of MSM in PLWHA. It has previously been reported that HIV-infected MSM are at an increased risk of contracting syphilis. A survey carried out in 14 cities throughout China reported that the prevalence of syphilis was $6.7 \%$ in MSM adults, ${ }^{34}$ which was lower than that reported in Tianjin ${ }^{35}(11 \%, 2016)$. A total of $11.4 \%$ of HIV-infected MSMs with confirmed syphilis was reported in Guangzhou, China. ${ }^{36}$ Homosexual transmission was the main route of HIV infection in Tianjin, and the higher proportion of MSMs with unfixed sex partner may be responsible for the higher rate of HIV/ syphilis coinfection. In the present study, at baseline, syphilis infections among HIV-infected MSM accounted for $65.3 \%$ of the total HIV infected individuals. The proportion of co-infected MSM decreased from $80 \%$ to $30 \%$ from 2009 to 2019, which was inconsistent with a previous study. ${ }^{1,33,35,37-39}$ Effective education in the correct use of latex condoms may be responsible for this phenomenon.

This study had several limitations. First, retrospective studies are subject to innate bias. Second, the study was carried out in a single health center and the conclusions drawn from this study may not be fully representative of what would be seen throughout China. Our findings should, therefore, be confirmed by further studies. Third, HIV viral load at baseline was not included in the medical records and we could not exclude the effect of baseline viral load on immune response. Fourth, HCV and HBV seroconversion after ART initiation were not recorded, and thus, the effect of such seroconversion was not considered. Fifth, assessments of viral load were performed yearly or twice per year during the follow-up period, and is a weakness that could not be addressed as it is the protocol used in our hospital. Sixth, this is the only study that analyzed factors associated with changes in HIV viral loads and CD4 cell count during ART in HIV/syphilis coinfected patients. Further studies of the effects of genital ulcers on HIV-1 transmission and HCV, HBV seroconversion on the immunology and virology of PLWHA are warranted.

\section{Conclusions}

In conclusion, this study reveals the relationship between $\mathrm{HIV} /$ syphilis co-infection and responses to ART during a seven-year follow-up period. The higher virologic failure rate in co-infected patients argues for close monitoring of ART treatment effects and HIV disease progression in this population, especially in reinfected patients or patients with recurrent infection. The attenuated immunologic responses seen here support earlier initiation of ART in $\mathrm{HIV} /$ syphilis co-infected patients to optimize their intreatment $\mathrm{CD}^{+} \mathrm{T}$ cell counts. RPR titers should also be monitored in HIV/syphilis co-infected patients, especially in patients with high RPR titers, even if these individuals respond well to treatment for syphilis. Further research is needed to determine whether early treatment of syphilis in 
A

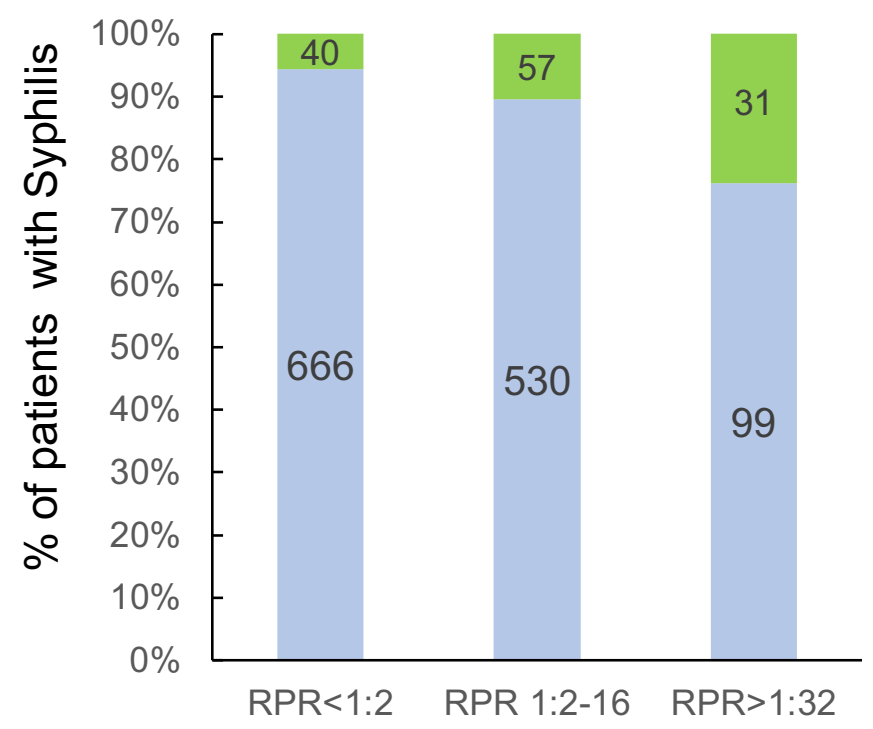

Recurrent or

Re-infected with syphilis

syphilis

\section{B}

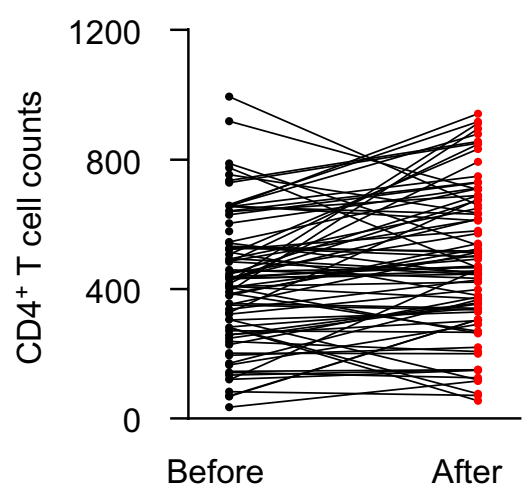

C

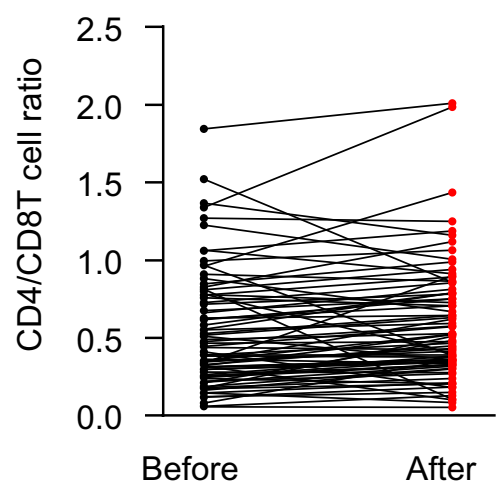

Figure 5 (A) RPR titers at baseline in recurrent syphilis or reinfection patients; (B) CD4 ${ }^{+} \mathrm{T}$ cell counts during the one-year periods before or after the diagnosis of recurrent syphilis or reinfection in patients receiving ART; (C) CD4/CD8 ratios during the one-year periods before or after recurrent syphilis or reinfection diagnosis in patients receiving ART.

HIV/syphilis co-infected patients would be associated with improved responses to ART and better clinical outcomes.

\section{Abbreviations}

HIV, human immunodeficiency virus; AIDS, acquired immune deficiency syndrome; ART, anti-retroviral therapy; MSM, men who have sex with men; HR, hazard ratio; RPR, rapid plasma reagin; BMI, body mass index; PLWHA, people living with HIV/AIDS, STI, sexually transmitted infection, AOR, adjusted odds ratio, OIS, opportunistic infections.

\section{Acknowledgments}

We would like to express our highest appreciation for the fantastic work of the health care providers. Their dedication, 
in terms of diagnosis, nursing and treatment of HIV/AIDS patients at Tianjin Second People's Hospital, is highly valued. This work was supported by grants from the Thirteen-fifth Key Project (No. 2018ZX10302104-001) and the Internal Project of Tianjin Second People's Hospital (No. YS0017). The fund providers played no role in study design, data collection or analysis, the decision to publish, or the preparation of the manuscript. We thank Dr Silvere D Zaongo and International Science Editing (http://www.internationalscien ceediting.com) for editing this manuscript.

\section{Disclosure}

All authors agreed to publication of this article and declare no conflicts of interest.

\section{References}

1. Sarigül F, Sayan M, Inan D, et al. Current status of HIV/ AIDS-syphilis co-infections: a retrospective multicentre study. Cent Eur J Public Health. 2019;27(3):223-228. doi:10.21101/cejph.a5467

2. Mata-Marín JA, Sandoval-Sánchez JJ, Huerta-García G, et al. Prevalence of antibodies against Treponema pallidum among HIV-positive patients in a tertiary care hospital in Mexico. Int J STD AIDS. 2015;26(2):81-85. doi:10.1177/0956462414530888

3. Zhou Y, Li D, Lu D, Ruan Y, Qi X, Gao G. Prevalence of HIV and syphilis infection among men who have sex with men in China: a meta-analysis. BioMed Res Int. 2014;2014:620431.

4. Ning TL, Guo Y, Zheng MN, et al. The characteristics of recent HIV-1 infection and associated factors in Tianjin. Chin $J$ Preven Med. 2019;53(3):323-326.

5. Lynn WA, Lightman S. Syphilis and HIV: a dangerous combination. Lancet Infect Dis. 2004;4(7):456-466. doi:10.1016/S1473-3099(04) 01061-8

6. Ghanem KG, Erbelding EJ, Wiener ZS, Rompalo AM. Serological response to syphilis treatment in HIV-positive and HIV-negative patients attending sexually transmitted diseases clinics. Sex Transm Infect. 2007;83(2):97-101. doi:10.1136/sti.2006.021402

7. Marra CM, Deutsch R, Collier AC, et al. Neurocognitive impairment in HIV-infected individuals with previous syphilis. Int J STD AIDS. 2013;24(5):351-355. doi:10.1177/0956462412472827

8. Rekart ML, Ndifon W, Brunham RC, et al. A double-edged sword: does highly active antiretroviral therapy contribute to syphilis incidence by impairing immunity to Treponema pallidum? Sex Transm Infect. 2017;93(5):374-378. doi:10.1136/sextrans-2016-052870

9. Chesson HW, Pinkerton SD, Irwin KL, Rein D, Kassler WJ. New HIV cases attributable to syphilis in the USA: estimates from a simplified transmission model. AIDS (London, England). 1999;13 (11):1387-1396. doi:10.1097/00002030-199907300-00017

10. Palacios R, Jiménez-Oñate F, Aguilar M, et al. Impact of syphilis infection on HIV viral load and CD4 cell counts in HIV-infected patients. J Acquir Immune Deficien Syndr. 2007;44(3):356-359. doi:10.1097/QAI.0b013e31802ea4c6

11. Kofoed K, Gerstoft J, Mathiesen LR, Benfield T. Syphilis and human immunodeficiency virus (HIV)-1 coinfection: influence on CD4 T-cell count, HIV-1 viral load, and treatment response. Sex Transm Dis. 2006;33(3):143-148. doi:10.1097/01.olq.0000187262.56820.c0

12. Buchacz K, Patel P, Taylor M, et al. Syphilis increases HIV viral load and decreases CD4 cell counts in HIV-infected patients with new syphilis infections. AIDS (London, England). 2004;18 (15):2075-2079. doi:10.1097/00002030-200410210-00012
13. Taylor MM, Li WY, Skinner J, Mickey T. Viral loads among young HIV-infected men with early syphilis. J Int Assoc Provid AIDS Care. 2014;13(6):501-505. doi:10.1177/2325957414536229

14. Sadiq ST, McSorley J, Copas AJ, et al. The effects of early syphilis on CD4 counts and HIV-1 RNA viral loads in blood and semen. Sex Transm Infect. 2005;81(5):380-385. doi:10.1136/sti.2004.012914

15. Saag MS, Gandhi RT, Hoy JF, et al. Antiretroviral drugs for treatment and prevention of HIV infection in adults: 2020 recommendations of the International Antiviral Society-USA panel. JAMA. 2020;324 (16):1651-1669. doi:10.1001/jama.2020.17025

16. Hiransuthikul A, Pattanachaiwit S, Teeratakulpisarn N, et al. High subsequent and recurrent sexually transmitted infection prevalence among newly diagnosed HIV-positive Thai men who have sex with men and transgender women in the test and treat cohort. Int J STD AIDS. 2019;30(2):140-146. doi:10.1177/0956462418799213

17. World Health Organization. WHO Case Definitions of HIV for Surveillance and Revised Clinical Staging and Immunological Classification of HIV-Related Disease in Adults and Children. World Health Organization; 2007.

18. AIDS and Heptitis C Professional Group SoID, Chinese Medical Association, Chinese center for Disease Control and Prevention. Chinese guidelines for diagnosis and treatment of HIV/AIDS (2018). Chin J Int Med. 2008;57:1-18.

19. Chen CM. Overview of obesity in Mainland China. Obesity Rev. 2008;9(Suppl 1):14-21. doi:10.1111/j.1467-789X.2007.00433.x

20. Jin MJ, Chen BB, Mao YY, et al. Prevalence of overweight and obesity and their associations with socioeconomic status in a rural Han Chinese adult population. PLoS One. 2013;8(11):e79946. doi:10.1371/journal.pone.0079946

21. Bruno G, Saracino A, Monno L, Angarano G. The revival of an "old" marker: CD4/CD8 ratio. AIDS Rev. 2017;19(2):81-88.

22. Serrano-Villar S, Moreno S, Fuentes-Ferrer M, et al. The CD4: CD8ratio is associated with markers of age-associated disease in virally suppressed HIV-infected patients with immunological recovery. HIV Med. 2014;15(1):40-49. doi:10.1111/hiv.12081

23. Serrano-Villar S, Sainz T, Lee SA, et al. HIV-infected individuals with low CD4/CD8 ratio despite effective antiretroviral therapy exhibit altered $\mathrm{T}$ cell subsets, heightened $\mathrm{CD} 8+\mathrm{T}$ cell activation, and increased risk of non-AIDS morbidity and mortality. PLoS Pathog. 2014;10(5):e1004078. doi:10.1371/journal.ppat.1004078

24. McBride JA, Striker R, Coyne CB. Imbalance in the game of T cells: what can the CD4/CD8 T-cell ratio tell us about HIV and health? PLoS Pathog. 2017;13(11):e1006624. doi:10.1371/journal. ppat. 1006624

25. Riddler SA, Aga E, Bosch RJ, et al. Continued slow decay of the residual plasma viremia level in HIV-1-infected adults receiving long-term antiretroviral therapy. $J$ Infect Dis. 2016;213(4):556-560. doi:10.1093/infdis/jiv433

26. Luo X, Zhang X, Gan L, et al. The outer membrane protein Tp92 of Treponema pallidum induces human mononuclear cell death and IL-8 secretion. J Cell Mol Med. 2018;22(12):6039-6054.

27. Seña AC, Wolff M, Martin DH, et al. Predictors of serological cure and Serofast State after treatment in HIV-negative persons with early syphilis. Clin Infect Dis. 2011;53(11):1092-1099. doi:10.1093/cid/cir671

28. Spagnuolo V, Poli A, Galli L, et al. Incidence and predictors of serological treatment response in early and late syphilis among people living with HIV. Open Forum Infect Dis. 2019;6(1):ofy324. doi:10.1093/ofid/ofy324

29. Wang Z, Liu L, Shen YZ, et al. The clinical and laboratory features of neurosyphilis in HIV-infected patients: a retrospective study in 92 patients. Medicine. 2018;97(9):e0078. doi:10.1097/ MD.0000000000010078

30. Badie BM, Yavari Z, Esmaeeli S. Prevalence survey of infection with Treponema pallidum among HIV-positive patients in Tehran. Asian Pac J Trop Biomed. 2013;3(4):334-336. doi:10.1016/S22211691(13)60074-X 
31. Tsachouridou O, Skoura L, Christaki E, et al. Syphilis on the rise: a prolonged syphilis outbreak among HIV-infected patients in Northern Greece. Germs. 2016;6(3):83-90. doi:10.11599/ germs.2016.1093

32. Spornraft-Ragaller P, Schmitt J, Stephan V, Boashie U, Beissert S. Characteristics and coinfection with syphilis in newly HIV-infected patients at the University Hospital Dresden 1987-2012. JDDG. 2014;12(8):707-716.

33. Dai W, Luo Z, Xu R, et al. Prevalence of HIV and syphilis co-infection and associated factors among non-commercial men who have sex with men attending a sexually transmitted disease clinic in Shenzhen, China. BMC Infect Dis. 2017;17(1):86. doi:10.1186/s12879-017-2187-1

34. Qi J, Zhang D, Fu X, et al. Syphilis infection and its high risk factors among men who have sex with men by different recruitment channels. Zhonghua Yu Fang Yi Xue Za Zhi. 2015;49(5):399-404.

35. Zhang DC, Wu ZY, Scott SR. Behavior characteristics and HIV/ syphilis infection status among MSM students in Tianjin from 2012 to 2016. Chin J AIDS STD. 2018;24(1):58-61.
36. Huang L, Cheng W, Han Z, et al. Syphilis infection does not affect immunodeficiency progression in HIV-infected men who have sex with men in China. Int $J$ STD AIDS. 2020;31(5):488-496. doi: $10.1177 / 0956462419860618$

37. Huang Q, Li Q, Li Y, et al. Prevalence of HIV infection and syphilis, sexual behaviors and awareness of HIV/AIDS related knowledge among men who have sex with men in China: a Meta-analysis of data collected from 2010 to 2013. Zhonghua Liuxingbingxue Zazhi. 2015;36(11):1297-1304.

38. Fan S, Yang Z, Hou F, et al. HIV and syphilis and sexual risk behaviours among men who have sex with men attending university in China: a systematic review and meta-analysis. Sex Health. 2019;16 (6):554-565. doi:10.1071/SH18231

39. Chow EP, Wilson DP, Zhang L. HIV and syphilis co-infection increasing among men who have sex with men in China: a systematic review and meta-analysis. PLoS One. 2011;6(8): e22768. doi:10.1371/journal.pone.0022768

\section{Publish your work in this journal}

Infection and Drug Resistance is an international, peer-reviewed openaccess journal that focuses on the optimal treatment of infection (bacterial, fungal and viral) and the development and institution of preventive strategies to minimize the development and spread of resistance. The journal is specifically concerned with the epidemiology of antibiotic resistance and the mechanisms of resistance development and diffusion in both hospitals and the community. The manuscript management system is completely online and includes a very quick and fair peerreview system, which is all easy to use. Visit http://www.dovepress.com/ testimonials.php to read real quotes from published authors. 